КОНЪЮГИРОВАННАЯ ЛИНОЛЕВАЯ КИСЛОТА И ЕЁ ВЛИЯНИЕ НА УВЕЛИЧЕНИЕ СКОРОСТИ СНИЖЕНИЯ ВЕСА И УЛУЧШЕНИЕ АНТРОПОМЕТРИЧЕСКИХ ПОКАЗАТЕЛЕЙ У МОЛОДЫХ МУЖЧИН С ИЗБЫТОЧНОЙ МАССОЙ ТЕЛА НА ПРОТЯЖЕНИИ КОМПЛЕКСНОЙ ПРОГРАММЫ ПО СНИЖЕНИЮ ВЕСА

(с) Аккила С.С.

Университет Мустансирия, Багдад, Ирак

ИСХОДНАЯ ИНФОРМАЦИЯ: С увеличением распространенности ожирения в Ираке возрастает приверженность к физической активности среди молодых людей. В то же время увеличение количества предлагаемых пищевых добавок способствует улучшению физической формы и позволяет избавиться от лишнего веса. Конъюгированная линолевая кислота (CLA - conjugated linoleic acid) часто используется для такой цели.

ЦЕЛЬ: Текущее исследование было предназначено в целях апробирования действия конъюгированной линолевой кислоты и ее способности действительно ускорять снижение веса в сочетании с диетой и умеренными физическими упражнениями, а также исследование их сочетанного эффекта в отношении атропометрических показателей при ожирении.

ОБЪЕКТЫ И МЕТОДЫ: 60 молодых мужчин добровольцев были включены в исследование, которое предполагало использование диеты с пониженным содержанием углеводов и жиров, а также комплекс высокоинтенсивных умеренных физических упражнений с временным интервалом (HIIT - high intensity interval exercise). 40 из них получали конъюгированную линолевую кислоту (1000 мг/день) в течение 8 недель, в то время как оставшиеся 20 участников составили контрольную группу. Индекс массы тела (body mass index - BMI), отношение окружности талии к окружности бедер (waist/hip ratio - WHR), сагиттальный абдоминальный диаметр (sagittal abdominal diameter - SAD), процент содержания жира в организме (body adiposity index - BAl) были зарегистрированы в начале, после 4 и 8 недель исследования.

РЕЗУЛЬТАТЫ: Группа участников, которая употребляла конъюгированную линолевую кислоту, показала существенно большее снижение всех показателей в конце исследования. В контрольной группе снижение показателей было значительным, но такие показатели как BMI и SAD оказались менее сниженными, чем в основной группе, однако WHR и BAI оставались аналогичными. Процент изменений отличался между 1-ой и 2-ой частями исследования, также показаны статистические различия между двумя группами и для различных показателей. Наиболее важным явилось то, что SAD снизился значительно быстрее за время первых 4-х недель исследования, указывая на преимущественное уменьшение висцерального жира. Напротив, WHR снизился за время 2-ой половины изучения, больше за счет снижения абдоминального жирового депо.

ОБСУЖДЕНИЕ: Независимо от механизма действиям CLA, ускорение действия в снижении веса происходит только в комбинации с диетой и физической активностью. Это также соответствует преимущественно естественному снижению более метаболически активного висцерального жира до перехода к снижению подкожного жира. Является ли данная пищевая добавка эффективной или нет, требует дальнейшего изучения.

КЛЮЧЕВЫЕ СЛОВА: избыточная масса тела, ожирение, конъюгированная линолевая кислота, индекс массы тела, соотношение окружности талии к окружности бедер, сагиттальный абдоминальный диаметр, процент содержания жира в организме, висцеральный жир, подкожный жир.

\title{
CONJUGATED LINOLEIC ACID ACCELERATES WEIGHT LOSS AND IMPROVES ANTHROPOMETRIC MEASURES IN OVERWEIGHT YOUNG ADULT MALES DURING WEIGHT LOSS PROGRAM
}

(c) Akkila S.S.

Al-Mustansiriya University, Baghdad, Iraq

BACKGROUND: With the increasing prevalence of obesity in Iraq, an increasing trend towards better physical fitness has emerged among young adults. At the same time, an increasing number of dietary supplements is offered to improve fitness and shed the extra weight. Conjugated linoleic acid (CLA) is commonly used for such purpose.

AIM: The current study was designed to test if CLA can actually accelerate weight loss when combined with dieting and moderate exercise and to examine its effect on anthropometric measures of obesity. 
SUBJECTS \& METHODS: Sixty young males enrolled willingly in the study and were put on a low-carbohydrate low-fat diet and moderate high intensity interval exercise (HIIT) program. Forty of them were given CLA (1000 mg/ day) for 8 weeks while the remaining twenty served as control group. Body mass index (BMI), waist/hip ratio (WHR), sagittal abdominal diameter (SAD) and body adiposity index (BAI) were recorded as baseline and again after 4 and 8 weeks.

RESULTS: The CLA-treated group showed significantly greater reduction in all four measures by the end of the study. The control group had significant but less decrease in BMI \& SAD but not in WHR or BAI. The percent change difference between the first and second four weeks of the study also showed statistical difference among the two groups and for different measures. The most significant finding was that SAD dropped more rapidly during the first 4 weeks of the study indicating a preferential loss of visceral fat. Oppositely, WHR dropped during the second half of the study indicating loss of more abdominal fat stores.

DISCUSSION: Regardless of the mode of action of CLA, its accelerative effect in weight loss is augmented by the combination with dieting and physical activity. It also corresponds to the natural body preference to losing the more metabolically active visceral fat prior to switching to the loss of subcutaneous fat. Whether or not this effect is sustainable requires further study.

KEYWORDS: overweight, obesity, conjugated linoleic acid, body mass index, waist/hip ratio, sagittal abdominal diameter, body adiposity index, visceral fat, subcutaneous fat

\section{ВВЕДЕНИЕ}

В 2014г. в Ираке доля лиц с ожирением (ИМТ $\geq 30)$ составила $21.2 \%$, с избыточной массой тела (ИМТ $\geq 25)$ более 50 \% [1]. Однако, за последние несколько лет наблюдается увеличение интереса к физической активности среди подростков и молодых мужчин Ирака, что влечет за собой с такой целью резкое увеличение количества спортивных залов и клубов. Многие из данных заведений обеспечены множеством пищевых добавок, которые, предположительно, повышают метаболизм, ускоряют снижение веса и улучшают наращивание мышечной массы. Одной из общеупотребимых пищевых добавок является конъюгированная линолевая кислота. Она включает группу позиционных и геометрических (цис- и транс-) изомеров жирной кислоты, соединенных между собой двойной связью [2]. Несколько механизмов действия конъюгированной линолевой кислоты были предложены в различных исследованиях, включая супрессию энергии всасывания, липогенеза и адипогенеза, усиление энергии расхода и апоптоз преадипоцитов [3]. Таким образом, многие исследования показали благоприятные структурные эффекты конъюгированной линолевой кислоты в отношении различных видов животных, другие исследования показали только незначительное положительное влияние на организм человека. Различные дозированные системы, типы изомеров и животные модели в сравнении с несколькими данными исследований на человеке могут объяснить наблюдаемые отличия в изучении действия конъюгированной линолевой кислоты [5].

\section{ЦЕЛЬ}

Текущее исследование было запланировано для определения влияния конъюгированной линолевой кислоты как пищевой добавки на антропометрические показатели у молодых мужчин Ирака с избыточной массой тела, участвующих в комплексной программе по снижению массы тела, включая диету и физические упражнения умеренной интенсивности.

\section{ОБЪЕКТЫ И МЕТОДЫ}

\section{Условия}

Исследование выполнялось в двух спортивных залах Аль-Карского района (Багдад, Ирак) на протяжении 8 недель (сентябрь 2016г., октябрь 2016 г.).

\section{Этические вопросы}

Все участники были подробно проинформированы о подготовке исследования и его цели, а также все подписали информированное согласие. Измерения тела регистрировались с соблюдением конфиденциальности. Вопросы конфиденциальности ни разу не были скомпрометированы.

Определение случаев, включенных в исследование, критерии включения и исключения. Исследование включало 60 мужчин молодого возраста с избыточной массой тела (ИМТ=25-29,9), которые занимались в вышеупомянутых спортивных залах с целью снижения веса. Никто из исследуемых не принимал препаратов, влияющих на метаболизм; не имел эндокринных и хронических заболеваний, ограничивающих физическую активность или нарушающих обмен веществ в организме. Все участники были согласны с программой исследования, включая диету и физические упражнения.

\section{Выборка и рандомизация}

60 молодых мужчин (24,7士1,78 лет) 23-26 лет были включены в исследование. Участников выбирали в произвольном порядке каждый 2-ой день в течение 2 недель, после их согласия и готовности участвовать.

Исходный и окончательный результаты. Первоначальный результат состоял в том, чтобы определить, влияет ли сочетанное применение конъюгированной линолевой кислоты совместно с диетой и физическими упражнениями умеренной интенсивности на снижение веса в сравнении с использованием только диеты и умеренной физической нагрузки. Окончательный результат был основан на определении изменений в висцеральном и подкожном жировых депо за определенный период времени с использованием антропометрических показателей у лиц с ожирением. 


\section{Расчет калорий}

Индивидуальный базальный метаболический коэффициент был рассчитан с помощью уравнения Mifflin-St. Jeor (Mifflin-St.Jeor equation) [6]:

$B M R=(10 \times$ вес в кг) $+(6.25 \times$ рост в см $)-(5 \times$ возраст в годаx) +5

С того момента, когда обследуемые стали заниматься физическими упражнениями (умеренные высокоинтенсивные кардио упражнения 3-5 дней в неделю), увеличилась ежедневная потребность в калориях в 1,55 раз. (Ежедневное потребление калорий = $\mathrm{BMR} \times 1,55)$ [7]. Потребность участников в калориях составила 2838,1土98,06 SD (Ккал/день). Потребность была скорректирована после 4-х недель с учетом изменений массы тела.

\section{Диета}

Всем участникам была предложена модифицированная диета с пониженным содержанием углеводов и жиров, на 500 ккал меньше, чем ежедневная потребность [8]. Питание включало 30\% углеводов, 25\% жиров (<7\% насыщенные жиры), 35\% белков, 10\% свежих фруктов и овощей. Участникам дан список продуктов, которые следовало исключить (например, конфеты, масло, сыр, фаст фуд), употреблять ежедневно (например, свежая зелень, фрукты, цельное зерно, тощее мясо), дробное питание 5-6 раз/день.

\section{Физические упражнения}

Участники исследования были обучены высокоинтенсивным интервальным тренировкам (НIIT) 3-5 дней/ неделю с использованием беговой дорожки. Занятия состояли из 5 циклов, продолжительностью 25 минут. Каждый цикл (5 минут) включал 3 минуты быстрой ходьбы (5-6 км/час) с последующей 2-минутной пробежкой (9,5 км/час). Выполняемым занятиям предшествовала 3-минутная разминка, 3-минутный период охлаждения с целью предупреждения мышечных спазмов.

Исследуемые группы и антропометрические измерения: испытуемые участники были поделены на 2 группы. 1-я группа (40 человек) ежедневно однократно принимала 1000 мг конъюгированной линолевой кислоты (Nutrex Research Inc., USA) в течение 8 недель. В исследовании использовались 10-цис- и 12-транс- изомеры. 2-я группа (20 человек) соблюдала только диетические рекомендации и выполняла физические упражнения на протяжении 8 недель.

Все антропометрические показатели и расчеты записывались непосредственно перед исследованием (первоначальные данные), в конце 4-ой недели и в конце 8-ой недели. Измерения проводились через 4 часа после приема пищи и были выполнены в соответствии с протоколом Всемирной организации здравоохранения [9]. Расчеты проводились согласно установленным эпидемиологическим стандартам [10].

Измерение веса проводилось без обуви и одежды, только в шортах, при помощи электронных весов (ANYSCALES ${ }^{\circledR}$ TCS-200-RT, Australia) с точностью до 100 г. Весы оснащены ростомером, который используется для измерения роста стоя (в анатомическом положении) с точностью до см. Окружности талии и бедер снимаются в положении стоя только в шортах. Окружность талии измеряется в средней точке между нижним краем последнего ребра и верхней частью подвздошного гребня с помощью сантиметровой ленты. Окружность бедер измеряется в самой широкой части ягодиц сантиметровой лентой параллельно горизонтальной поверхности [11]. Каждый показатель повторялся дважды; если данные измерений оказывались с разницей в пределах 1 см, выводилось среднее значение. Если различие между 2-мя показателями превышало 1 см, регистрировались оба значения. Сагиттальный абдоминальный диаметр (SAD) измерялся в наклонном положении с согнутыми коленями при помощи сагиттометра [9], раздвижной перекладины калипера с линейкой (SkynDex ${ }^{\circledR}$, USA, d: 0.1 $\mathrm{cm})$. Размер снимался на уровне талии, нижняя ручка калипера устанавливалась снизу, верхняя на уровне живота сверху, размеры регистрировались в конце выдоха с осью калипера в вертикальном положении. Величина SAD в норме $<25$ см [12].

Индекс массы тела рассчитывался путем деления веса, кг на рост, м в квадрате кг/м²). Величина в пределах 18,5-24,9 относится к норме, 25-29,9 - к избыточной массе тела [13]. Отношение окружности талии к окружности бедер вычисляется путем деления окружности талии на окружность бедер в см. Величина более чем 0,9 считается высокой[14].

Коэффициент содержания жира (BAI) в организме рассчитывается с помощью формулы:

BAI = Окружность бедер $($ м) $/[\operatorname{Pocm}($ м) $x \sqrt{ }(\operatorname{Bec}($ м) $)]$ - 18 [15].

Предпочтительно достичь величины 8\%-21\% [16].

Статистический анализ: Для анализа полученных данных используется программа SPSS software version 18.0. Переменные и множества как правило распределяются и представляются как количество процентов для категорий переменных, среднее \pm стандартное отклонение (SD) для числовых переменных. Независимый обучающий т-тест использовался для сравнения возможностей групп.

\section{РЕЗУЛЬТАТЫ}

Ни у кого из обследуемых, получавших конъюгированную линолевую кислоту, не отмечалось побочных эффектов за период исследования. В таблице 1 показана частота распределения показателей с отклонением от нормы у молодых мужчин с избыточной массой тела как в группе принимающих конъюгированную линолевую кислоту, так и в контрольной группе в начале и в конце исследования. В конце исследования выявлено резкое снижение BMI и SAD в обоих группах, но снижение оказалось больше в группе принимающих конъюгированную линолевую кислоту. WHR и BAI существенно снизились в CLA группе, что не наблюдалось в контрольной группе.

В таблице 2 показаны изменения антропометрических показателей в динамике у лиц с ожирением в основной и контрольной группах. BMI и SAD стабильно снижались в обоих группах, но общее число изменений оказалось значительно больше в основной группе (рис. 1). В основной группе в начале исследования было отмечено повышение показателя WHR, схожие изменения наблюдались и в контрольной группе, но в конце исследования, что свидетельствует о более быстром темпе снижения. Показатель BAІ варьировал значительно боль- 
Таблица 1. Начальная и конечная частота распределения отклонений антропометрических показателей у лиц с ожирением в основной и контрольной группах

\begin{tabular}{lcccc}
\hline \multirow{2}{*}{\multicolumn{1}{c}{ Величина }} & \multicolumn{3}{c}{ Группа } \\
\cline { 2 - 5 } & \multicolumn{2}{c}{ основная (частота \%) } & \multicolumn{2}{c}{ контроль (частота \%) } \\
\cline { 2 - 5 } & Начало & Окончание & Начало & Окончание \\
\hline Избыточный вес BMI & $100 \%$ & $22.5 \%^{* *}$ & $100 \%$ & $35 \%^{* *}$ \\
Высокий WHR & $97.5 \%$ & $37.5 \%^{* *}$ & $95 \%$ & $90 \%$ \\
Высокий SAD & $75 \%$ & $5 \%^{* *}$ & $65 \%$ & $5 \% *$ \\
Высокий BAI & $90 \%$ & $22,5 \%^{* *}$ & $95 \%$ & $85 \%$ \\
\hline
\end{tabular}

BMI: Индекс Массы Тела, WHR: отношение окружности талии к обхвату бедер, SAD: Сагиттальный Абдоминальный Диаметр, BAI: процент содержания жира в организме, **: Высокая статистическая значимость с р-величиной $<0,01$.

Таблица 2. Динамика изменений антропометрических показателей у лиц с ожирением в основной и контрольной группах. (Представлены средние числовые значения \pm SD)

\begin{tabular}{lcccccc}
\hline \multirow{2}{*}{ Величина } & \multicolumn{3}{c}{ Основная группа } & \multicolumn{3}{c}{ Контрольная группа } \\
\cline { 2 - 7 } & Начало & 4-я неделя & 8-я неделя & Начало & 4-я неделя & 8-я неделя \\
\hline BMI & $27,46 \pm 1,08$ & $26,04 \pm 1,13$ & $24,45 \pm 1,01$ & $26,97 \pm 0,76$ & $25,91 \pm 0,80$ & $24,94 \pm 0,84$ \\
WHR & $0,969 \pm 0,08^{*}$ & $0,958 \pm 0,07$ & $0,927 \pm 0,08$ & $0,941 \pm 0,002$ & $0,938 \pm 0,002$ & $0,927 \pm 0,002$ \\
SAD & $27,32 \pm 3,47$ & $23,28 \pm 2,29$ & $20,69 \pm 4,88$ & $26,32 \pm 3,17$ & $24,31 \pm 4,11$ & $21,66 \pm 9,43$ \\
BAI & $24,48 \pm 2,27$ & $21,83 \pm 2,8^{* *}$ & $20,21 \pm 3,09^{* *}$ & $24,85 \pm 2,14$ & $23,54 \pm 2,01$ & $22,64 \pm 4,32$ \\
\hline
\end{tabular}

BMI: Индекс Массы Тела, WHR: отношение окружности талии к обхвату бедер, SAD: Сагиттальный Абдоминальный Диаметр, BAI: процент содержания жира в организме, ${ }^{*}$ : статистическая значимость с р-величиной $<0.05,{ }^{* *}$ : Высокая статистическая значимость с р-величиной $<0.01$.

ше в основной группе как на 4-ой, так и на 8-ой неделях исследования.

На рисунке 1 показан процент изменений атропометрических показателей у лиц с ожирением в динамике на 4-ой и 8-ой неделе исследования. Для всех параметров и в любые сроки исследования процент изменений в основной группе был значимо больше, чем в контрольной группе, за исключением показателя SAD после 2-ой половины 4-ой недели, который изменялся в обеих группах с аналогичной скоростью.

Суммарная доля по показателям BMI и BAI в обеих группах существенно не отличалась между 1-ой и 2-ой частью исследования. Значение WHR значительно больше снизилось во 2-ой половине исследования. Уменьшение показателя SAD оказалось больше в группе обследуемых, принимавших конъюгированную линолевую кислоту во время первых 4-х недель исследования. Напротив, этот же показатель в контрольной группе значительно снизился за время вторых 4-х недель исследования.

\section{ОБСУЖДЕНИЕ}

Текущее исследование было запланировано согласно правилам снижения веса, что приводило к ограничению энергетических субстратов, путем сокращения их потребления и увеличения расхода энергии [17]. Уменьшение антропометрических показателей у лиц с ожирением оказалось более значимым в группе молодых мужчин, дополнительно принимавших конъюгированную линолевую кислоту, что свидетельствует о благоприятном действии лекарственных препаратов на снижение веса. Согласно другим исследованиям, выполненным на человеке, конъюгированная линолевая кислота использовалась в качестве отдельной пищевой добавки [18], либо в составе пищевых

First 4 weeks $\%$ change

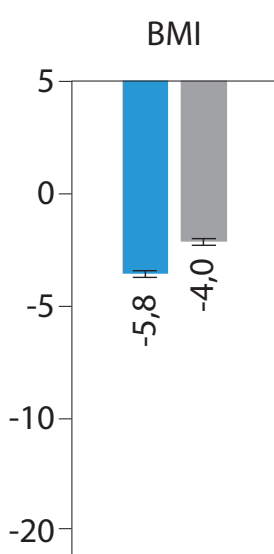

BAI

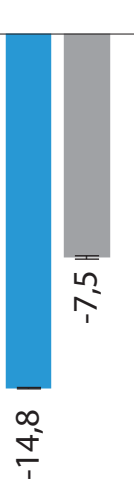

Second 4 weeks \% change

BMI WHR SAD BAI

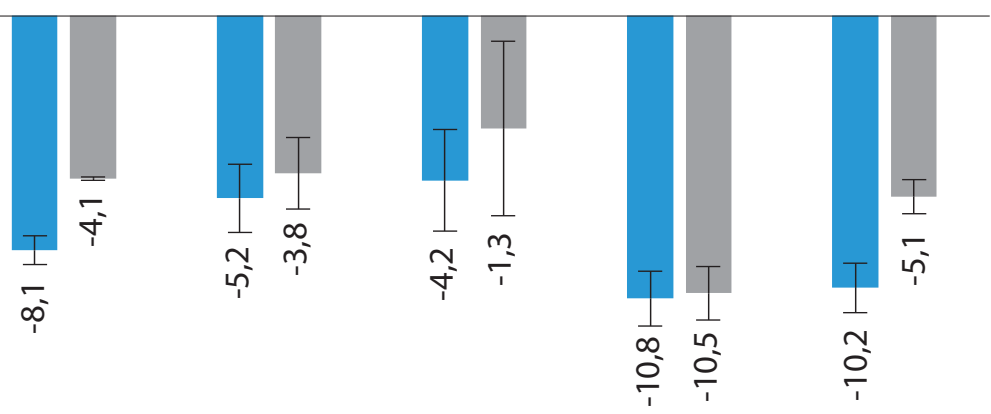

CLA Control

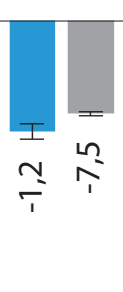

$$
\underset{1}{\simeq}
$$

Рис. 1. Процент изменений антропометрических показателей у лиц с ожирением в основной и контрольной группах после 4 и 8 недель исследования.

Представленные данные = средняя величина, погрешности = SD. BMI: Индекс Массы Тела, WHR: отношение окружности талии к окружности бедер, SAD: Сагиттальный Абдоминальный Диаметр, BAl: процент содержания жира в организме. *: статистическая значимость с р-величиной <0,05. 
продуктов (например, молочные продукты) [19], эффективно влияющих на снижение веса, при условии ограничения калорийности рациона. Согласно различным исследованиям, вопрос о точном механизме действия конъюгированной линолевой кислоты до сих пор остается спорным. Некоторые исследования предполагают, что конъюгированная линолевая кислота уменьшает потребление пищи и сокращает жировые отложения с увеличением или без расхода энергии [3]. Другие ученые утверждают, что данное вещество снижает созревание преадипоцитов и увеличивает апоптоз зрелых адипоцитов [20]. Это новое объяснение наиболее подходит для текущего исследования, так как предварительно рассчитаны ограничения калорийности рациона и контроль режима питания.

Изменения антропометрических показателей у лиц с ожирением в основной группе данного исследования были значительно больше, чем в контрольной группе, за исключением индекса массы тела, который был одинаковым в обоих группах.

Недостатком индекса массы тела является неспособность разграничить тощую массу тела и жировые депо [21, 22] и пр.

B настоящем исследовании показатели SAD, WHR и BAI существенно не уменьшились в контрольной группе, кроме BMI. Кроме того, снижение BMI за время второй половины исследования происходило медленнее в обеих группах, возможно, по причине наращивания мышечной массы вторично, на фоне физических упражнений умеренной интенсивности. Становится очевидным, что распределение жира является более важным детерминирующим фактором, связанным с метаболическими нарушениями при ожирении, чем степень избыточного веса, измеряемая по BMI. SAD, являющийся показателем висцеральной абдоминальной ткани (VAT), основан на том, что подкожная ткань варьирует в измерениях калипера в зависимости от воздействия силы тяжести [23]. С другой стороны, значение WHR рассчитывается с помощью измерения окружности талии, которая в свою очередь зависит от VAT и SAT [24]. Предыдущие исследования по снижению веса доказали, что наблюдается преимущественное снижение от VAT к SAT в сочетании с умеренным ограничением в питании [25].

B настоящем исследовании снижение SAD предшествовало снижению WHR. Уменьшение VAT, о чем свидетельствует снижение SAD, произошло намного быстрее и при более высоких показателях в основной группе во время первых 4-х недель исследования, но в контроль- ной группе данный показатель увеличивался постепенно, достигнув максимума во время вторых 2-х недель исследования. Это свидетельствует об ускоренном эффекте CLA в снижении VAT. Такой процесс может быть связан с тем, что VAT более метаболически активен, чем SAT, возможно, благодаря большей клеточности, васкуляризации, более лучшей иннервации и чувствительности к некоторым гормонам, подобно глюкокортикоидам (выброс которых стимулируется диетой и физической нагрузкой) [26]. SAD продолжал значительно уменьшаться в контрольной группе во время вторых 4-х недель исследования возможно потому, что уменьшение жира преимущественно все же связано с VAT. Данный факт подтверждается снижением WHR, который в обоих группах был незначительным первые 4 недели исследования, но увеличился за вторые 4 недели, в наибольшей степени в основной группе обследуемых лиц.

Действие CLA на WHR и SAT заключается в их снижении, что может быть связано с влиянием физических упражнений. Физические тренировки могут существенно уменьшить VAT у лиц с избыточной массой тела даже без ограничений в питании [27], но это требует более длительной работы, воздействующей на абдоминальный SAT, так как SAT адаптационно реагирует в ответ на тренировочные упражнения [28].

CLA может изменить такой адаптационный ответ после ичерпанности преимущественного снижения VAT. Возможные механизмы включают увеличение физической активности и постфизическое окисление жиров и увеличение катехоламиндуцированного липолиза [29].

\section{Выводы}

В то время, как ограничения в питании и увеличение физической активности дают удовлетворительные результаты по снижению массы тела и коррекции антропометрических показателей у мужчин с избыточной массой тела, CLA в качестве пищевой добавки может ускорить данные результаты. Существует ли подобный эффект при высоких степенях ожирения и сохраняется ли он с течением времени - требует дальнейшего изучения.

\section{КОНФЛИКТ ИНТЕРЕСОВ}

Автор объявляет об отсутствии конфликтов интересов или специального финансирования для текущего изучения. Автор также благодарен за ощутимую помощъ и сотрудничество Mr. Rami M. Khalid.

Перевод Сенюшкина Евгения.

\section{СПИСОК ЛИТЕРАТУРЫ | REFERENCES}

The World Fact Book. [Internet] CIA. Central Intelligence Agency. [22 June 2014. Retrieved: 1 June 2017]. Available from: https://www.cia. gov/library/publications/the-world-factbook/fields/2228.html. Koba K, Yanagita T. Health benefits of conjugated linoleic acid (CLA). Obes. Res. Clin. Pract. 2014;8(6):e525-e532. doi: 10.1016/j.orcp.2013.10.001

3. Kennedy A, Martinez K, Schmidt S, et al. Antiobesity mechanisms of action of conjugated linoleic acid. The Journal of Nutritional Biochemistry. 2010;21(3):171-179. doi: 10.1016/j.jnutbio.2009.08.003.

4. Wahle KWJ, Heys SD, Rotondo D. Conjugated linoleic acids: are they beneficial or detrimental to health? Prog. Lipid Res. 2004;43(6):553-587. doi: 10.1016/j.plipres.2004.08.002.
5. McCrorie TA, Keaveney EM, Wallace JMW, et al. Human health effects of conjugated linoleic acid from milk and supplements. Nutrition Research Reviews. 2012;24(02):206-227. doi: $10.1017 / \mathrm{s} 0954422411000114$

6. Mifflin MD, St Jeor ST, Hill LA, et al. A new predictive equation for resting energy expenditure in healthy individuals. The American Journal of Clinical Nutrition. 1990;51 (2):241-247. doi: 10.1093/ajcn/51.2.241.

7. Bazzano LA, Hu T, Reynolds K, et al. Effects of Low-Carbohydrate and Low-Fat Diets. Ann. Intern. Med. 2014;161(5):309. doi: 10.7326/m14-0180.

8. Zwetsloot KA, Nieman DC, Knab A, et al. Effect of 4 weeks of high-intensity interval training on exercise performance and markers 
of inflammation and oxidative stress. The FASEB Journal. 2017;31(1_ supplement):839.831-839.831. doi: 10.1096/fasebj.31.1_supplement.839.1.

9. Organization WH. WHO STEPS surveillance manual: the WHO STEPwise approach to chronic disease risk factor surveillance. 2005.

10. Vuga M. Conceptual review of issues with practical abdominal obesity measures. Section on Statistics in Epidemiology-JSM. 2009.

11. Organization WH. Waist circumference and waist-hip ratio: report of a WHO expert consultation, Geneva, 8-11 December 2008. 2011.

12. Riserus U, Arnlov J, Brismar K, et al. Sagittal Abdominal Diameter Is a Strong Anthropometric Marker of Insulin Resistance and Hyperproinsulinemia in Obese Men. Diabetes Care. 2004;27(8):2041-2046. doi: 10.2337/diacare.27.8.2041.

13. Organization WH. BMI Classification. Global Database on Body Mass Index. 2012.

14. Pouliot M-C, Després J-P, Lemieux S, et al. Waist circumference and abdominal sagittal diameter: Best simple anthropometric indexes of abdominal visceral adipose tissue accumulation and related cardiovascular risk in men and women. The American Journal of Cardiology. 1994;73(7):460-468. doi: 10.1016/0002-9149(94)90676-9.

15. Bergman RN, Stefanovski D, Buchanan TA, et al. A Better Index of Body Adiposity. Obesity. 2011;19(5):1083-1089. doi: 10.1038/oby.2011.38

16. Belarmino G, Horie LM, Sala PC, et al. Body adiposity index performance in estimating body fat in a sample of severely obese Brazilian patients. Nutrition journal. 2015;14(1) doi: 10.1186/s12937-015-0119-8.

17. Almirall D, Nahum-Shani I, Sherwood NE, Murphy SA. Introduction to SMART designs for the development of adaptive interventions: with application to weight loss research. Transl. Behav. Med. 2014;4(3):260274. doi: 10.1007/s13142-014-0265-0

18. Saboktakin M, Zamani E. Determination of the effect of cla supplementation on weight loss in people under treatment regimen. Nutrition and food sciences research. 2014;1 (1):237-237.

19. Stonehouse W, Wycherley T, Luscombe-Marsh N, et al. Dairy Intake Enhances Body Weight and Composition Changes during Energy Restriction in 18-50-Year-Old Adults-A Meta-Analysis of Randomized Controlled Trials. Nutrients. 2016;8(12):394. doi: 10.3390/nu8070394.
20. Wang YW, Jones PJH. Conjugated linoleic acid and obesity control: efficacy and mechanisms. Int. J. Obes. 2004:28(8):941-955. doi: 10.1038/sj.ijo.0802641.

21. Müller M, Kahn HS, Gu Q, et al. Population Distribution of the Sagittal Abdominal Diameter (SAD) from a Representative Sample of US Adults: Comparison of SAD, Waist Circumference and Body Mass Index for Identifying Dysglycemia. PLoS One. 2014;9(10):e108707. doi: 10.1371/journal.pone.0108707.

22. Finucane MM, Stevens GA, Cowan MJ, et al. National, regional, and global trends in body-mass index since 1980: systematic analysis of health examination surveys and epidemiological studies with 960 country-years and 9.1 million participants. The Lancet. 2011;377(9765):557-567. doi: 10.1016/s0140-6736(10)62037-5.

23. Yim JY, Kim D, Lim SH, et al. Sagittal Abdominal Diameter Is a Strong Anthropometric Measure of Visceral Adipose Tissue in the Asian General Population. Diabetes Care. 2010;33(12):2665-2670. doi: 10.2337/dc10-0606.

24. Shuster A, Patlas M, Pinthus JH, Mourtzakis M. The clinical importance of visceral adiposity: a critical review of methods for visceral adipose tissue analysis. The British Journal of Radiology. 2012;85(1009):1-10. doi: 10.1259/bjr/38447238.

25. Chaston TB, Dixon JB. Factors associated with percent change in visceral versus subcutaneous abdominal fat during weight loss: findings from a systematic review. Int. J. Obes. 2008;32(4):619-628. doi: 10.1038/sj.ijo.0803761.

26. Ibrahim MM. Subcutaneous and visceral adipose tissue: structural and functional differences. Obes. Rev. 2010;11(1):11-18. doi: 10.1111/j.1467-789X.2009.00623.x.

27. Votruba SB, Vissers D, Hens W, et al. The Effect of Exercise on Visceral Adipose Tissue in Overweight Adults: A Systematic Review and Meta-Analysis. PLoS One. 2013;8(2):e56415. doi: 10.1371/journal. pone. 0056415 .

28. Gollisch KSC, Brandauer J, Jessen N, et al. Effects of exercise training on subcutaneous and visceral adipose tissue in normal- and high-fat diet-fed rats. American Journal of Physiology-Endocrinology and Metabolism. 2009;297(2):E495-E504. doi: 10.1152/ajpendo.90424.2008.

29. Boutcher SH. High-Intensity Intermittent Exercise and Fat Loss. J. Obes. 2011;2011:1-10. doi: 10.1155/2011/868305.

\section{ИНФОРМАЦИЯ ОБ АВТОРАХ [AUTHORS INFO]}

Аккила Самех. [Sameh S. Akkila] адрес: Ирак, 14022, Багдад, ул. Палестины. [address: Palestine Street, 14022 Baghdad, Iraq]; ORCID: http://orcid.org/0000-0002-0407-0685; e-mail: samakkila@gmail.com

\section{ЦИТИРОВАТЬ:}

Аккила С.С.. Конъюгированная линолевая кислота и её влияние на увеличение скорости снижения веса и улучшение антропометрических показателей у молодых мужчин с избыточной массой тела на протяжении комплексной программы по снижению веса. // Ожирение и метаболизм. — 2018. - T.15. — №. 1 — C.19-24. doi: 10.14341/OMET2018119-24

\section{TO CITE THIS ARTICLE:}

Akkila SS. Conjugated linoleic acid accelerates weight loss and improves anthropometric measures in overweight young adult males during weight loss program. Obesity and metabolism. 2018;15(1):19-24. doi: 10.14341/OMET2018119-24 